\title{
Breast infarction during pregnancy and lactation: A case report
}

\author{
BING HAN ${ }^{1}$, HAIPENG ZHANG ${ }^{2}$, PENGLI JIANG ${ }^{1}$, CHAO ZHENG $^{1}$, LIRONG BI $^{3}$, LU LU $^{1}$ and ZHIMIN FAN ${ }^{1}$ \\ ${ }^{1}$ Division of Breast Surgery; ${ }^{2}$ Department of Obstetrics; ${ }^{3}$ Clinical Pathology Diagnosis Center, \\ The Bethune First Hospital, Jilin University, Changchun, Jilin 130021, P.R. China
}

Received September 25, 2014; Accepted June 26, 2015

DOI: 10.3892/etm.2015.2756

\begin{abstract}
Spontaneous infarction is a rare condition associated with the physiological hyperplasia of breast tissue associated with pregnancy and lactation. The causes of and mechanism underlying the occurrence of this complication remain largely unknown. The present study describes a rare case of breast infarction occurring during pregnancy and lactation in a 20-year-old woman. At 2 months of gestation, the patient noticed a soft and painless lump (size, $\sim 5 \times 4 \mathrm{~cm}$ ) in the right breast. The lump grew to eventually occupy the entire breast. The patient was hospitalized 1 month after delivery and underwent a mastectomy. Histopathological study of the resected breast tissue revealed that $90 \%$ of the breast tissue had undergone infarction, with the infarct located centrally, under the areola. Involution of the breast tissue and small focal hemorrhages were noted, along with acute or chronic inflammatory cell infiltration in the interstitial tissue. Some breast ducts showed cystic dilatation, while some small blood vessels showed dilatation and congestion. Postoperative recovery of the patient was uneventful. This was a case of breast infarction with irregular, high-grade fever. The findings of core-biopsy were inconclusive, which highlights the importance of Mammotome ${ }^{\mathrm{TM}}$ biopsy or surgical excision in the diagnosis in such cases.
\end{abstract}

\section{Introduction}

Breast infarction occurs as a sequela of benign breast lesions, such as fibroadenomas, or as a spontaneous change in healthy breast tissue typically during pregnancy and lactation (1). Spontaneous solitary infarction is very rare. Infarction of the breast tissue with hyperplasia related to pregnancy and/or lactation was first reported by Hasson and Pope in 1961 (2). Infarction typically presents as a palpable mass that may be painful and varies between soft and hard in consistency. It can be mistaken for a carcinoma due to the hard consistency of the

Correspondence to: Dr Zhimin Fan, Division of Breast Surgery, The Bethune First Hospital, Jilin University, 71 Xinmin Street, Changchun, Jilin 130021, P.R. China

E-mail: fanzhimn163@163.com

Key words: breast infarction, pregnancy and lactation lesion. Infarcts usually occur as a single lesion, localized to a fibroadenoma, or hyperplastic lactating breast tissue during the peripartum period; however, extensive, multifocal, bilateral mammary infarction is very rare (3). Localized infarction is most frequently observed in the third trimester of pregnancy or early postpartum period. In this report, a rare case of breast infarction occurring during pregnancy and lactation in a 20-year-old woman is presented.

\section{Case report}

A 20-year-old woman first noticed a painless lump $(\sim 5 \times 4 \mathrm{~cm}$ in size) in her right breast at 2 months of gestation. However, she did not seek medical attention for the lump. The lump gradually increased in size but remained painless. At term, she delivered a healthy, normal baby boy by cesarean section. During lactation, the patient noted that milk production in the right breast was less than that in the left one. Over a period of 1 month after delivery, the lump grew rapidly in size with marked discoloration of the overlying skin. The patient also had irregular, high-grade fever, with the body temperature rising to $39^{\circ} \mathrm{C}$. The patient was then admitted to hospital at 1 month postpartum. The patient had no history of taking any special medication. Clinical examination revealed a soft lump of size $18 \times 16 \mathrm{~cm}$ in her right breast with pigmentation and induration of the overlying skin (Fig. 1). A palpable lymph node $(\sim 2 \times 1.5 \mathrm{~cm})$ was identified in the right axilla of the patient. The patient had no history of trauma or use of oral contraceptives.

Color Doppler ultrasonography of the right breast revealed a well-circumscribed area of mixed echogenicity, with an approximate size of $199.7 \times 56.7 \mathrm{~mm}$; the heterogeneous echogenicity of the internal region of the mass suggested fluid content. Some lymph nodes with abnormal appearance were noted in the right axilla, with the maximum size of the lymph nodes being 19.0x12.1 mm (Fig. 2). Mammography of the right breast showed a regular, high-density mass shadow, with an approximate size of $11.0 \times 13.0 \mathrm{~cm}$ (Fig. 3). Normal mammary gland tissue could not be identified on the mammogram. No clear calcification was found within and around the lesion.

T1-weighted nuclear magnetic resonance imaging (NMRI) of the right breast revealed the presence of a mass of size $13.4 \times 10.9 \mathrm{~cm}$ showing heterogeneous signal intensity, with areas of isointensity, slight hyperintensity and hypointensity. A scan with edge enhancement showed a significant increase in heterogeneity. No abnormality in signal intensity was identified in the pectoralis. Soft tissue masses, with visible 
enhancement and diameter 0.4-1.4 cm, were also detected in the right axilla (Fig. 4).

The patient had unexplained, irregular high-grade fever, but no post-cesarean wound infection. Gynecological ultrasonography revealed that the depth of ascitic fluid posterior to the uterus was $11 \mathrm{~mm}$, which suggested that the possibility of postoperative infection was minimal. The results of blood culture tests were negative. Urine culture revealed the presence of Corynebacterium species at a concentration of $<10,000 \mathrm{cfu} / \mathrm{ml}$. The fever (temperature, $38^{\circ} \mathrm{C}$ ) persisted.

Core-needle biopsy was performed to establish the pathological diagnosis and revealed that the lump was composed of necrotic and unorganized material. No clear tumor lesions were found within and around the lump. At 5 days after admission, minimally invasive Mammotome ${ }^{\mathrm{TM}}$ biopsy was performed to further confirm the diagnosis; examination of the biopsied sample revealed fibroadenosis in the right breast, with lobular hyperplasia, tissue infarction and unorganized structure. Therefore, surgical treatment was considered.

Since there was no evidence of normal breast tissue in the right breast, we advised the patient to undergo mastectomy and prosthetic reconstruction. The patient underwent mastectomy but refused prosthetic reconstruction for financial reasons. Histopathological study of the resected breast tissue revealed that $90 \%$ of the breast tissue had undergone infarction and that the infarct was located centrally, under the areola. The breast tissue also exhibited tissue involution and small focal hemorrhages. The interstitial tissue showed infiltration of acute and chronic inflammatory cells. Some of the breast ducts showed cystic dilatation, and the formation of cysts with galactostasia. The nipple and milk ducts did not show any evident lesions, and the basal tissue of the breast did not show any evidence of infarction, with the exception of dilatation and congestion of small blood vessels to a certain extent. Postoperative recovery of the patient was good and uneventful, and the fever subsided following the surgical resection (Figs. 5 and 6). This study was approved by the ethics committee of the Bethune First Hospital, Jilin University (Jilin, China) and written informed consent was provided by the patient.

\section{Discussion}

Spontaneous breast infarction is a rare entity. Infarction of the breast tissue with hyperplasia associated with pregnancy and/or lactation was first reported by Hasson and Pope in 1961 (2). Infarction typically presents as a palpable mass that is sometimes painful and soft or hard in consistency. It can be mistaken for a carcinoma due to the hard consistency of the lesion. Infarcts usually occur as a single lesion, localized to a fibroadenoma, or hyperplastic lactating breast tissue during the peripartum period; however, extensive, multifocal, bilateral mammary infarction is very rare (3). Localized infarction is most frequently observed in the third trimester of pregnancy or early postpartum period. Breast infarction during pregnancy or lactation may also be mistaken for fibroadenoma (4). Clinically, fibroadenoma typically occurs in young women during pregnancy or lactation and presents as a firm, smooth, painless and easily palpable lump with a well-defined shape. These features are very similar to those of spontaneous infarction of the breast; therefore, it is very difficult to distinguish between the two lesions on the basis of clinical examination alone. Other terms used to describe breast infarction in the published literature are adenoma associated with pregnancy or lactation adenoma, drug-induced breast necrosis (5), and ischemic fat necrosis of the mammary region (6). In the present case, the results of pathologic examination revealed the presence of hyperplastic breast lobules and ischemic necrosis rather than adenomatous features. Thus, the distinction between infarction and lesions such as fibroadenoma may not be very difficult. However, during the diagnostic evaluation in this case, Bard-needle aspiration biopsy revealed that most tissues were necrotic, with loss of organized structure. The diagnosis of the lesions could not be definitively established by core-needle biopsy alone because the sample size collected was small; this highlights the importance of performing Mammotome $^{\mathrm{TM}}$ biopsy or surgery to confirm the diagnosis of breast infarction.

On admission, the patient had high-grade, irregular fever. No case of breast infarction accompanied by fever has been reported thus far in the literature (7). The fever could have been related to urinary tract infection, heat absorption of necrotic tissue, or infection associated with the lump. After the surgery, the patient's body temperature was restored to normal, and no signs of infection were noted in the lump tissue. This implies that the patient's fever was most probably caused by heat absorption from the necrotic tissue.

The etiology of breast infarction is yet to be conclusively established; however, proposed theories implicate vascular insufficiency and localized thrombosis. None of the cases of breast infarction reported thus far have shown any evidence of vascular disease or trauma. Another factor associated with the occurrence of breast infarction is the use of oral contraceptives (4). Certain medications are known to exacerbate fibrocystic disease or compromise breast vascularity, which in turn results in mass formation and necrosis; however, none of the patient's medications have been shown to lead to breast complications (3). In the present case, the pathological examination showed hyperplastic breast lobules and ischemic necrosis, but no evidence of malignancy. Therefore, a diagnosis of ischemic infarct was made. The pathological examination did not reveal any clear signs of venous thrombosis or vascular endothelial lesions. One of the possible explanations for the occurrence of breast infarction in this case is that a local artery may have been compressed during the lactation period, during which a rich blood supply is necessary. This compression could have led to ischemic infarction of the glandular tissue distant from the base of the breast. However, no signs of infarction were noted in the breast, skin and subcutaneous tissue because of their intact inherent blood circulation system. The functions of the mammary glands are controlled by the neuroendocrine system. Therefore, neuroendocrine disorders may lead to partial arterial spasm, insufficient blood supply, and tissue ischemia in the mammary glands. In the present case, the patient should have been in the lactation stage, but the resected breast tissue showed involution of the remaining breast tissue on histopathological examination, thereby indicating dysfunction of the lactation system. The results of the pathological examination showed that $90 \%$ of the infarct was located centrally, under the areola, and no evidence of infarction was noted in the peripheral areas of the breast, including 


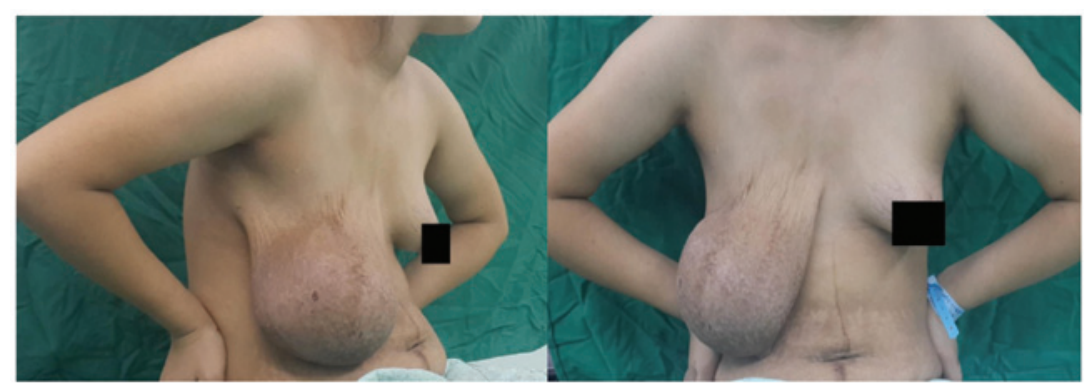

Figure 1. Clinical appearance of the right breast lump. The lump was $18 \times 16 \mathrm{~cm}$ in size, with soft consistency and pigmentation and induration of overlying skin.

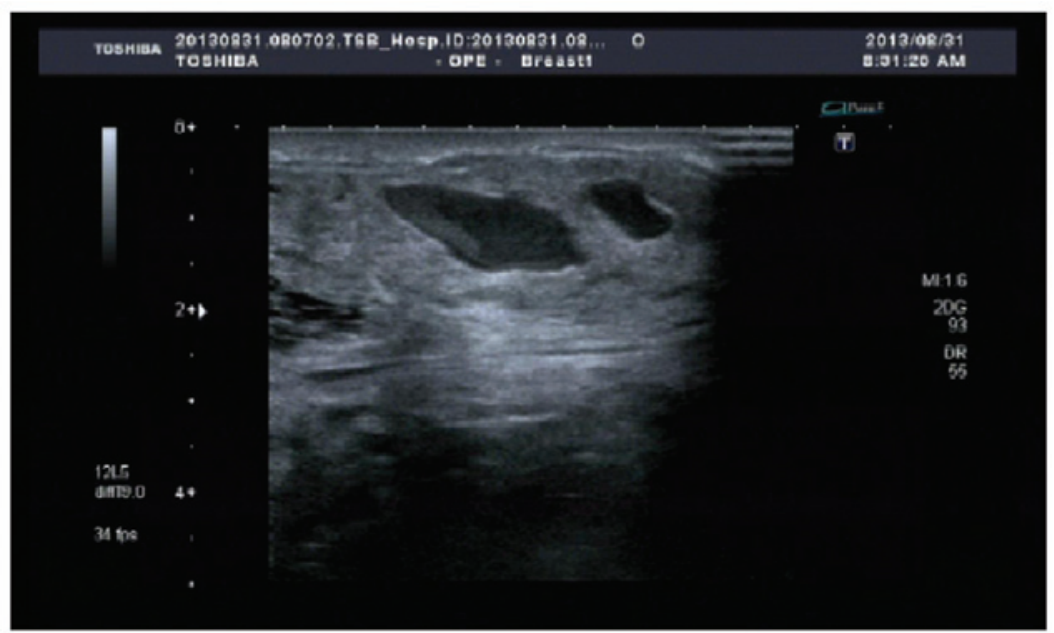

Figure 2. Ultrasonography of the right breast. Ultrasound examination of the right breast revealed a mixed echogenic mass, with an approximate size of 199.7x56.7 mm and a distinct border; the echogenicity within the border was heterogeneous, suggesting fluid content. The breast tissue exhibited involution.

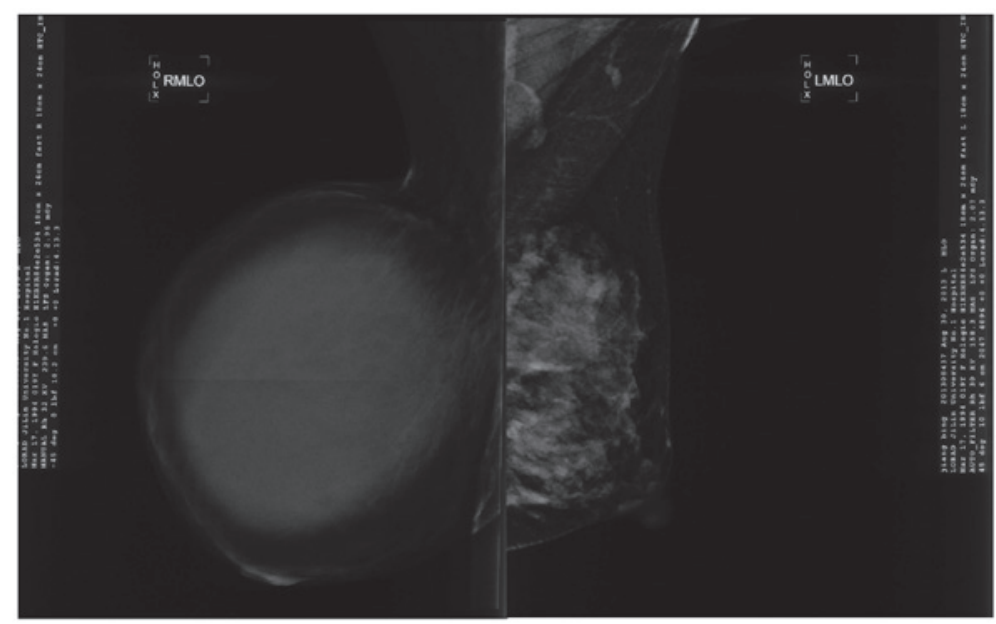

Figure 3. Mammography images of the right breast. Mammography revealed a regular high-density mass shadow in the right breast, with an approximate size of $11.0 \times 13.0 \mathrm{~cm}$. No distinct areas of calcification were noted within and around the lesion. The results were classified as breast imaging reporting and data system (BI-RADS) score of 4 .

the subcutaneous tissue and glandular tissue at the base of breast. This finding supports the possibility that the hyperplastic breast tissue underwent necrotic changes secondary to ischemia. However, these considerations are inadequate to explain why the infarction was unilateral rather than bilateral.

NMRI revealed linearly distributed areas of low signal intensity surrounding the mass. Enhanced edge scanning showed a significant enhancement of heterogeneity. The lesion was observed to be composed of mixed elements, including necrotic tissues and lactation-associated components. Non-enhanced NMRI revealed heterogeneous signals in the lesions; however, contrast-enhanced NMRI revealed pronounced heterogeneous signals in long and wedge-shaped regions that extended to the center of the lesions, which may 


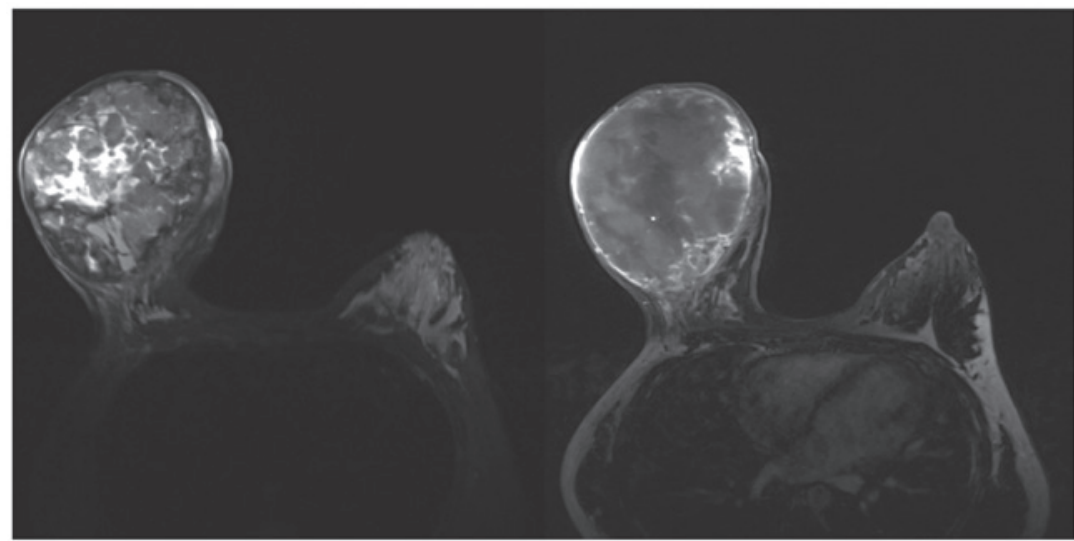

Figure 4. Findings of NMRI of the right breast. T1-weighted NMRI revealed a mass of heterogeneous signal intensity in right breast, with areas of isointensity, slight hyperintensity, and hypointensity. An enhanced edge scan image showed significant enhancement in the heterogeneous area. No abnormality in signal intensity was identified in the pectoralis. There were no abnormal signals evident in the left breast. NMRI, nuclear magnetic resonance imaging.

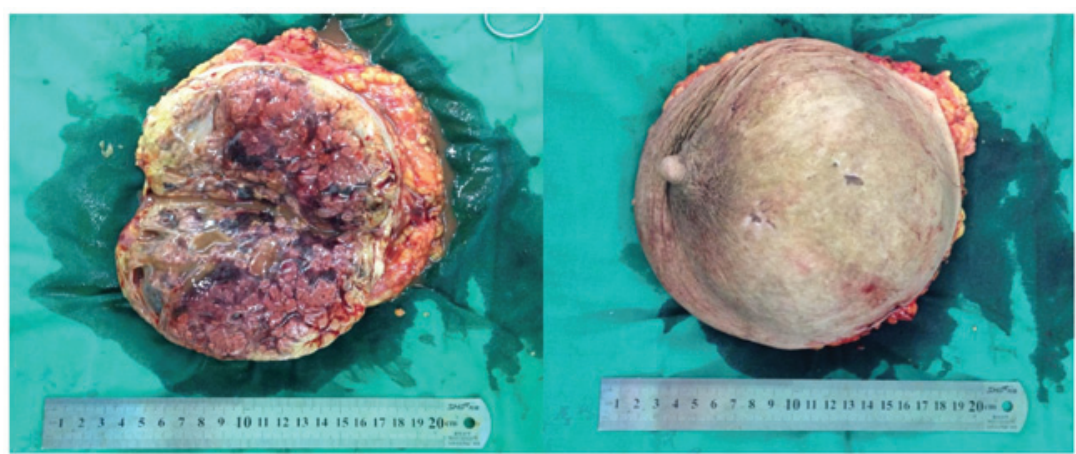

Figure 5. Appearance of the resected breast. The resected section showed a well-circumscribed mass measuring $22 \times 18 \times 9 \mathrm{~cm}$, attached by spindle-shaped skin $(\sim 18 \times 18 \mathrm{~cm}$ in size). The diameter of the nipple was $\sim 1.8 \mathrm{~cm}$. A huge lump measuring $17 \times 16 \times 7 \mathrm{~cm}$ occupied the entire breast. The lump showed a clear-cut distinction from the surrounding tissue, indicating the possibility of encapsulation. The cut section of the mass was pale brown in appearance. The mass was solid and hard in consistency. Localized cysts measuring $0.2-1.0 \mathrm{~cm}$ in diameter were detected within the mass.
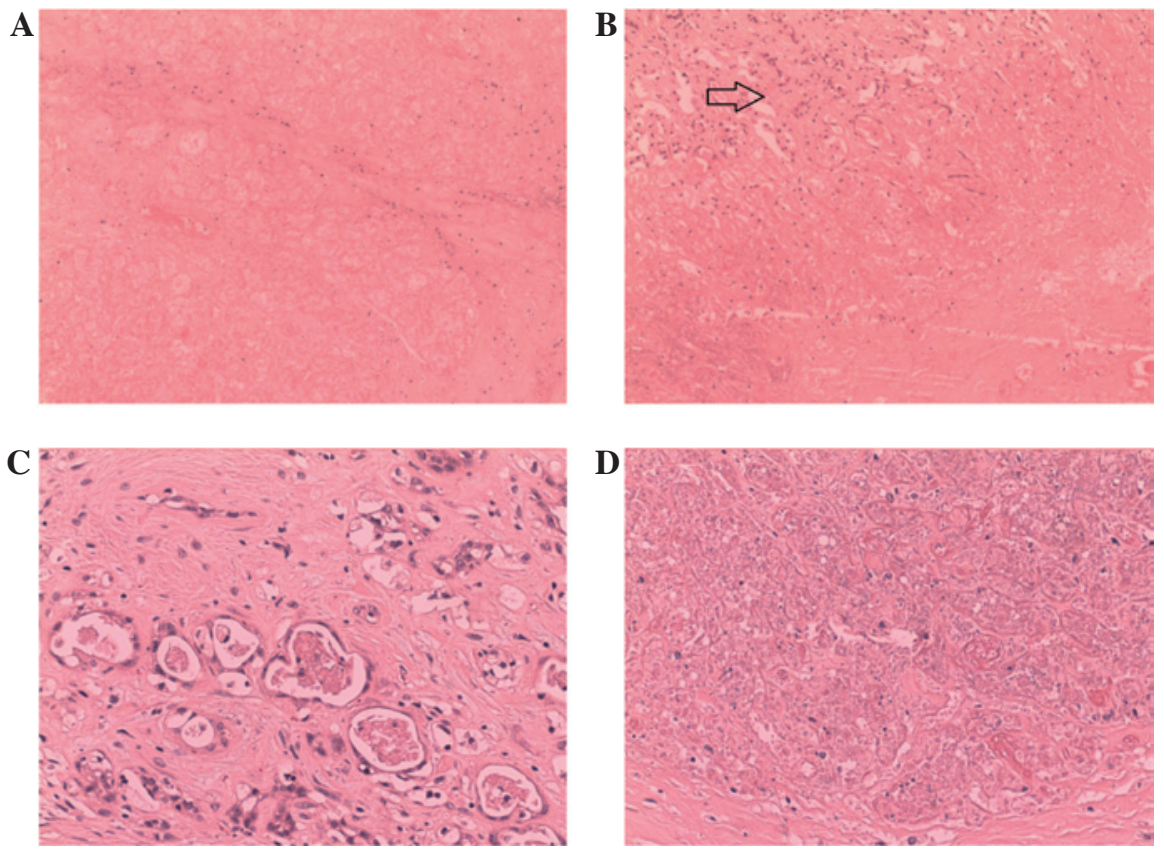

Figure 6. Clinicopathological examination of the resected breast tissue. The examination revealed that (A) $90 \%$ of the breast tissue on the right side was infarcted (H\&E staining; magnification, $\mathrm{x} 40$ ); (B) tissue surrounding the infarcted areas showed capillary proliferation, indicated by the arrow (H\&E staining; magnification, $\mathrm{x} 100$ ); (C) the remaining breast tissue showed dilatation of small ducts, with visible intraluminal secretions (H\&E staining, magnification, x100); (D) completely infarcted tissue with visible traces of milk and breast tissue infarction (H\&E staining; magnification, $\mathrm{x} 100$ ). H\&E, hematoxylin and eosin. 
represent granulation tissue growing into the infarcted area. Increases in the number and thickening of the arterial shadows were also observed around the lesion, which most probably reflects the compensatory dilatation and congestion of the collateral circulation around the lesion. It is possible that the surface of the lesion was covered in fibrous tissue that formed a capsule.

The majority of patients with breast infarction are treated with widespread local excision, which is often performed after pregnancy (7). In such cases, the period of hyperplasia of breast tissue ends at the time of the surgery, and therefore, no case of breast tissue remaining after local excision has been reported thus far. However, it remains to be determined whether widespread local excision is safe in patients with hyperplastic breast tissue. In the present case, the lump was too big (occupying the entire right breast) for wide local excision to be performed; therefore, mastectomy and prosthetic reconstruction was recommended. However, the patient refused prosthetic reconstruction because of financial constraints.

In conclusion, the present study describes a rare case of breast infarction associated with pregnancy and lactation presenting with irregular, high-grade fever. From experience in this case, it is suggested that in cases of breast infarction, the findings of core-needle biopsy should be cautiously interpreted when the sample size is small and that Mammotome ${ }^{\mathrm{TM}}$ biopsy or surgical excision should be performed to confirm the diagnosis. Widespread local excision is the treatment of choice for this condition.

\section{References}

1. Kavdia R and Kini U: WCAFTI: Worrisome cytologic alterations following tissue infarction; a mimicker of malignancy in breast cytology. Diagn Cytopathol 36: 586-588, 2008.

2. Hasson $\mathbf{J}$ and Pope $\mathrm{CH}$ : Mammary infarcts associated with pregnancy presenting as breast tumors. Surgery 49: 313-316, 1961.

3. Aggon AA, Eakin LO, Desimone N and Snyder JA: Extensive multifocal mammary infarction - a case report. Breast Care (Basel) 8: 143-145, 2013.

4. Skenderi F, Krakonja F and Vranic S: Infarcted fibroadenoma of the breast: Report of two new cases with review of the literature. Diagn Pathol 8: 38, 2013.

5. Davis CE Jr, Wiley WB and Faulconer RJ: Necrosis of the female breast complicating oral anticoagulant treatment. Ann Surg 175: 647-656, 1972.

6. Robitaille Y, Seemayer TA, Thelmo WL and Cumberlidge MC: Infarction of the mammary region mimicking carcinoma of the breast. Cancer 33: 1183-1189, 1974.

7. Behrndt VS, Barbakoff D, Askin FB and Brem RF: Infarcted lactating adenoma presenting as a rapidly enlarging breast mass. AJR Am J Roentgenol 173: 933-935, 1999. 